\title{
DISCURSOS ACERCA DE LA NEUTRALIDAD CON RELACIÓN AL ENCUADRE
}

Maria de Lourdes Benítez Psicóloga, IPU Magíster en Psicoterapia Psicoanalítica, IUPA Miembro de AUDEPP Correo electrónico: tanamalub@hotmail.com ORCID: 0000-0001-7450-5018 


\section{Resumen}

Este artículo surge de la tesis de maestría en Psicoterapia Psicoanalítica: Prácticas discursivas acerca de la neutralidad en la clínica actual. Estudio realizado en una asociación uruguaya de psicoterapia psicoanalítica (Benítez, 2019). A su vez, desarrolla el tema del encuadre, que formó parte de los núcleos conceptuales del marco teórico de la tesis y también del análisis. Dicha noción aparece en las respuestas de algunos participantes de la investigación cuando mencionan a qué asocian la neutralidad o en qué se expresa.

Este trabajo hace referencia a distintos aportes de autores con relación a la temática (Etchegoyen, 1986b; De Urtubey, 1999; Paciuk, 2002; Schkolnik, 2008; Alizade, 2002, etc.). Finalmente, cita algunos discursos de los psicoterapeutas consultados, quienes expresan que la neutralidad se vincula al encuadre; esos discursos se enlazan con el marco teórico a modo de análisis. Los entrevistados concuerdan con varios de los autores reseñados al referirse a los requisitos formales que regulan la sesión en la práctica psicoanalítica. Además, se destaca la importancia del establecimiento del encuadre y de su cumplimiento como valor protector para ambos protagonistas del proceso psicoterapéutico.

Palabras clave: neutralidad, discurso, encuadre, ética, técnica psicoanalítica.

\section{Discourses about neutrality in relation to the frame}

\section{Abstract}

This article arises from a Master's thesis in psychoanalytic psychotherapy: Discursive practices about neutrality in the current clinic. Study carried out in an Uruguayan association of psychoanalytic psychotherapy (Benitez, 2019). Also, the framing theme is developed, as it was part the conceptual nucleus of the thesis' theoretical framework. This notion appears in the responses of some research participants when they mention what they associate neutrality with or how it is expressed.

This work refers to different contributions from authors in relation to the topic (Etchegoyen, 1986b; De Urtubey, 1999; Paciuk, 2002; Schkolnik, 2008; Alizade, 2002, etc.). Finally, it cites discourses by the psychotherapists consulted, who express that neutrality is linked to the frame; these discourses are linked to the theoretical framework by way of analysis. The interviewees agree with several of the authors reviewed, referring to the formal requirements that regulate the session in psychoanalytic practice. In addition, the importance of establishing the frame and its compliance is highlighted, as a protective value for both protagonists of the psychotherapeutic process.

Keywords: neutrality, discours, frame, ethics, psychoanalytic technique. 
El psicoanálisis no es un sistema como los filosóficos, que parten de algunos conceptos básicos definidos con precisión y procuran apresar con ellos el universo todo, tras lo cual no resta espacio para nuevos descubrimientos y mejores intelecciones. Más bien adhiere a los hechos de su campo de trabajo, procura resolver los problemas inmediatos de observación, sigue tanteando y siempre dispuesto a corregir o variar sus doctrinas. Lo mismo que la química o la física, soporta que sus conceptos máximos no sean claros, que sus premisas sean provisionales, y espera del trabajo futuro su mejor precisión.

Sigmund Freud (1986a, p. 249)

Leí ese pasaje del maestro pocos días después de la entrega de mi tesis de maestría. Sentí que validaba la idea de revisitar uno de los principios de la técnica psicoanalítica y que, a su vez, era una invitación a continuar trabajando-investigando...

Pues bien, se comenzará por hacer referencia a la tesis de maestría en Psicoterapia Psicoanalítica, que se denomina Prácticas discursivas acerca de la neutralidad en la clínica actual. Estudio realizado en una asociación uruguaya de psicoterapia psicoanalítica (Benítez, 2019). Luego se abordará el tema del encuadre, que formó parte de los núcleos conceptuales del marco teórico y también del análisis, debido a que dicha noción surge en las respuestas de algunos participantes de la investigación al referirse a sus asociaciones con la neutralidad o bien a las expresiones de esta.

La tesis mencionada es una investigación cualitativa de carácter exploratorio y descriptivo. El marco teórico que se utilizó es el psicoanálisis 
freudiano en enlace con el psicoanálisis contemporáneo y la perspectiva constructivista de Charmaz (2006). En el capítulo que corresponde al marco teórico se presentan desarrollos teóricos sobre la neutralidad y otros conceptos vinculados a esta: la ética, el encuadre, la escuchaatención flotante, la abstinencia y la contratransferencia, entre otros.

En la literatura consultada no se encontró una posición única respecto a la neutralidad. Esta aparece como un tema complejo y controvertido entre las distintas corrientes psicoanalíticas. La neutralidad analítica se presenta discutida por varios autores (Greben y Lesser, 1976; Rosa, 1989; Renik, 1999; Schkolnik, 1999; etc.). Algunos expresan que fue superada por otros conceptos (Corbella y Botella, 2003; Ormart y Brunetti, 2006; etc.). Otros sugieren que puede ser utilizada más como guía que como regla y, aún así, la consideran útil (Eizirik, 2002; Shapiro, 2005). También están quienes entienden sus bondades y sus fallas (Bleichmar, 2013; García, 2002; Rappoport de Aisemberg, 2004: Thomä y Kächele, 1990). Finalmente, hallamos a los que la vinculan al encuadre (De Urtubey, 1999; Ormart, 2005; Muñiz, 2016).

Para el mencionado estudio se hicieron 18 entrevistas semiestructuradas a psicoterapeutas entre junio y noviembre de 2018. El número de participantes no se eligió, sino que se obtuvo al alcanzar el punto de saturación; en ese momento, el trabajo de campo se detuvo. La muestra estuvo representada por dos grupos: uno incluía psicoterapeutas con más de quince años en la asociación de psicoterapia psicoanalítica; el otro, psicoterapeutas con menos de cinco años. ${ }^{1}$ Ambos grupos estaban integrados por licenciados en Psicología, psicólogos, médicos y psiquiatras, de Montevideo y otros departamentos del país, ${ }^{2}$ que ejercían en forma

1 El criterio elegido acerca de los años de pertenencia a la asociación se fundamenta en el interés por explorar, en un espectro amplio, cómo inciden la formación y la experiencia en el discurso de los psicoterapeutas.

2 Dicha pauta muestral se debe a que se entiende que el ejercicio de la psicoterapia puede estar atravesado por diferencias en contextos de proximidad, ya que es posible la cercanía y el conocimiento entre el psicoterapeuta y el paciente. 
privada, para el Sistema Nacional Integrado de Salud o en instituciones. Además, se entrevistó a dos informantes calificados: la Dra. Fanny Schkolnik y el Mag. Abel Fernández.

Se realizó un análisis temático del contenido de las narrativas de los participantes enfocado en los sentidos que le concedían a la neutralidad, a su utilización en la práctica y a su incidencia en la eficacia de la psicoterapia psicoanalítica. La producción de información y el análisis se abordaron desde la perspectiva de los psicoterapeutas e informantes calificados consultados, pero se incluyó a su vez la voz de la investigadora, ya que el estudio se planteó como un encuentro dialógico en el que los tres participaron con su conocimiento y experiencia. De esa forma, se llegó a resultados que trascienden las propias palabras de los consultados.

Finalmente, se señala que la regla se asocia a una neutralidad caricaturizada como ortodoxa, ligada a la distancia, a lo utópico. Se distingue que se cuenta con ideologías que atraviesan la posición neutral. Sin embargo, al proponer la conceptualización de neutralidad del Diccionario de psicoanálisis, de Laplanche y Pontalis (1996), esta adquirió otros sentidos: se la vinculó al encuadre, a la ética, a la escucha y a la abstinencia del analista, lo que permite el ejercicio bien comprendido de la función analítica. A su vez, se considera que el término neutralidad no parece el más apropiado para tal noción. Entre los psicoterapeutas que ejercen en contextos de mayor proximidad no hubo acuerdo sobre si la cercanía con el paciente altera la neutralidad. Entre las consideraciones de los psicoterapeutas de más de quince años y las de aquellos de menos de cinco no se observaron diferencias sustanciales en relación a los tópicos planteados para comprender qué piensan del principio de neutralidad y de su aplicación en la clínica actual. Se confirmó la vigencia de la neutralidad y que su utilización colabora en la eficacia de la psicoterapia psicoanalítica (Benítez, 2019). 
Como ya se indicó, el marco teórico al que se recurrió es el psicoanálisis freudiano en enlace con el psicoanálisis contemporáneo. El encuadre psicoanalítico formó parte de uno de los núcleos conceptuales de dicho apartado.

En relación a ese término, Schroeder (2010) plantea que la palabra encuadre no aparece en los textos de Freud, así como tampoco está presente en el Diccionario de psicoanálisis, de Laplanche y Pontalis (1996). Schroeder expone que Freud se refiere en forma implícita en sus escritos técnicos a ciertas reglas generales que nos acercan a la noción de encuadre, lo cual se aprecia con claridad en su texto Sobre la iniciación del tratamiento (Freud, 1986c).

Vale señalar que el significado del verbo encuadrar tiene distintas acepciones: 'encerrar en un marco o cuadro', 'encajar, ajustar dentro de otra cosa', 'determinar los límites de algo, incluyéndolo en un esquema u organización' (Real Academia Española, 2021) y también es el acto de establecer los límites dentro de los cuales un acontecimiento tiene lugar o cobra sentido.

Ahora bien, en relación al proceso psicoterapéutico, la noción de encuadre ocupa un lugar destacado en el seno del método psicoanalítico. Según Paciuk (2002), al parecer fue Winnicott (s. f.) quien hizo las primeras alusiones a este concepto al hablar del setting, al que definió como «la suma de todos los detalles de la técnica» (s. p.). Luego, Bleger (1967) se refirió al encuadre al escribir Psicoanálisis del encuadre psicoanalítico, texto que se volvió un clásico de la temática. Según este autor, un proceso solo puede ser investigado cuando se mantienen las mismas constantes, es decir que el encuadre son las constantes en que se da el proceso.

$\mathrm{Al}$ igual que Bleger, Braun de Bagnulo et al. (1991) exponen que el encuadre abarca el rol del analista, el conjunto de factores espaciotemporales y parte de la técnica, que comprende el establecimiento de horarios, honorarios, vacaciones, etcétera. 
Paciuk (2002) puntualiza que entre los analistas generalmente se usa el nombre encuadre para lo que regula la conducta en la sesión y agrega que es un concepto que difiere del significado de setting que define Winnicott. Para el autor, en la práctica psicoanalítica se denomina encuadre al «conjunto relativamente reglado y estable de procedimientos y conceptos» (Paciuk, 2002, p. 38). Las normas del encuadre son propuestas por el psicoterapeuta al paciente y de esa manera se establece un contrato de trabajo. Sin dudas, las alteraciones al encuadre hechas por el paciente serán motivo de análisis; es lo invariante de este lo que permite se puedan analizar esas rupturas o los cambios que en él se introducen. En ese sentido, muestra su valor como norma que se acepta o se desconoce. Según Bleger (1967), en la situación analítica, cuando se dan las rupturas al encuadre, se lo tiende a mantener o restablecer al utilizar la interpretación. ${ }^{3}$

Paciuk (2002) distingue el encuadre ideal, que es el que exponen los textos, del encuadre real, que es el que aplica cada analista en cada situación. Este autor, al igual que Etchegoyen (1986a), va más allá del contenido del encuadre y su valor como norma, y hace referencia a la actitud mental del analista, la cual debe de estar atravesada por principios éticos que permiten el adecuado trabajo terapéutico. Sin embargo, De Urtubey (1999) plantea que los elementos del encuadre son varios y que debe ser invariable, lo cual hace referencia a los aspectos formales.

Para Schkolnik (2008), «El encuadre cumple una función de liberar y a la vez poner límites al funcionamiento en la sesión del paciente y el analista», y agrega más tarde «por eso, importa establecer un contrato en el que se expliciten: el número y duración de las sesiones, la frecuencia, la modalidad de pago, las fechas de vacaciones, etc., para la contención

3 ¿Qué sucede cuando el psicoterapeuta realiza alteraciones al encuadre? Para Bleger (1967), las rupturas al encuadre producen grietas por las que se introduce la realidad, el mundo fantasma del paciente queda sin depositario. Por otra parte, ¿no será imprescindible analizar nuestra contratransferencia antes de instaurar un cambio técnico? 
de lo que circula libremente» (p. 35). Pero la autora sostiene que las características de los elementos del encuadre pueden variar dadas las diversas situaciones en que se da la tarea analítica (por ejemplo, puede ser necesario hacer cambios en la frecuencia o en los honorarios acordados). Sin embargo, enfatiza que los límites siempre están, ya que establecen un continente y a la vez crean una necesaria tercerización.

A su vez, De Urtubey (1999) señala que otras condiciones son necesarias para que se mantenga el encuadre: formulación de la regla fundamental, neutralidad benevolente para el terapeuta, frustración, contenencia y solicitud hacia el paciente. Esta autora hace referencia explícitamente a la neutralidad, que considera parte del encuadre, y señala que «La neutralidad es un proceso de control del yo, sin el cual la distancia requerida para la toma de conciencia quedaría eliminada» (De Urtubey, 1999, p. 54). Se puede pensar que, con su planteo sobre la frustración como condición esencial para que se conserve el encuadre, hace referencia de forma implícita a Freud (1986c), quien proponía que hay que rehusar las satisfacciones a las cuales el paciente más aspira.

De Urtubey (1999) entiende por contenencia:

la instalación y luego el desarrollo de una confianza recíproca, cada uno en su rol, en un ambiente de trabajo, más bien agradable, donde la lucha contra la destructividad y la desobjetivación [...] son llevadas por los mejores medios y en tiempo útil. (p. 60)

Más tarde, la autora explica:

La solicitud es una fuerza importante y activa, capaz de neutralizar y dominar el afecto, en la transferencia y en la contratransferencia, de la agresión y de la autoagresión. Implica la consciencia de la importancia de las pulsiones destructoras y autodestructoras del paciente, del 
desarrollo posible de estas pulsiones en el analista, del límite de los esfuerzos terapéuticos con cada paciente y de la esperanza, sin certeza, que en la lucha triunfaremos. (p. 64)

De otra manera, Ormart (2005) refiere que la neutralidad forma parte de los elementos del encuadre y así lo señala: «La neutralidad en la historia del psicoanálisis postfreudiano adquirió el estatuto de antídoto a la contratransferencia. La neutralidad se volvió uno de los elementos centrales del encuadre, necesario para la aplicación de la técnica psicoanalítica» (p. 137). Por su parte, Alizade (2002) realiza una diferenciación entre el encuadre externo y el encuadre interno. El primero corresponde a los aspectos formales del encuadre y el segundo atañe al dispositivo de trabajo psicoanalítico en la mente del analista. No obstante, Giovannetti de Freitas (2005) aborda otro aspecto al ocuparse del encuadre:

Los pacientes que hoy aparecen, debido a vivir en un mundo en que no hay más fronteras y en que la idea o concepto de permanencia están sustituidos por el de velocidad y aceleración del tiempo, no pueden ser presentados al encuadre clásico del análisis, bajo peligro de que no se constituya ningún análisis. En nuestros días el analista tiene la función central de ir construyendo, con cada paciente, un encuadre posible para que el análisis se constituya. (p. 261)

Giovannetti de Freitas (2005) hace una reflexión interesante acerca de cómo inciden en el espacio analítico los tiempos que corren. Es innegable que con algunos pacientes se tiene que reconsiderar algunas cuestiones del encuadre, pero sin dejar de tener en cuenta su esencia, valor técnico y ético.

Como se ha señalado anteriormente, en la información recogida la neutralidad se relacionó con el encuadre. Así lo expresaron algunos de 
los psicoterapeutas psicoanalíticos consultados: «lo asocio al encuadre [...] tener una cuestión como óptima, un manejo adecuado, que tenga relación con la transferencia, con la contratransferencia, con el encuadre» (Entrevistada 2, comunicación personal, 12 de junio, 2018) ${ }^{4} \mathrm{y}$ «La neutralidad se expresa en el encuadre» (Entrevistado 3, comunicación personal, 19 de junio, 2018). ${ }^{5}$

Ahora bien, los psicoterapeutas pueden estar planteando dos posibilidades. Por un lado, se pueden estar refiriendo a los requisitos formales que regulan la sesión en la práctica psicoanalítica o, por otro, pueden estar aludiendo al encuadre como condición a ser internalizada y que tiene que ver con la actitud analítica.

Al decir de Alizade (2002 y apud Edelman et al., 2009), los aspectos formales atañen al encuadre externo y la actitud analítica es eso que tiene que estar en forma indispensable para que un tratamiento sea considerado como psicoanalítico. Para esta autora, el encuadre interno es el dispositivo de trabajo psicoanalítico en la mente del analista, es lo invisible y está sobreentendido en la observancia de las reglas de atención flotante, asociación libre, abstinencia ${ }^{6}$ y en el análisis del analista. Además, Alizade (apud Edelman et al., 2009) incluye en el encuadre interno la capacidad de empatía y de escucha, así como también la permeabilidad del analista a su propio inconsciente y al del paciente, y el ejercicio de la creatividad en el arte de la cura.

4 Licenciada en Psicología con menos de cinco años en la Asociación, que ejerce para el Sistema Nacional Integrado de Salud y en clínica privada.

5 Psicoterapeuta y médico psiquiatra con más de quince años en la Asociación, que se desempeña en el ámbito privado.

6 ¿Por qué no añadir la neutralidad analítica? No está pensada para ser utilizada por un observador preocupado por guardar una distancia que le permita ser objetivo, sino como posición que se asume durante el tratamiento y que refiere al decir de Laplanche y Pontalis (1996): «debe ser neutral en cuanto a sus valores religiosos, morales, sociales, es decir, no dirigir la cura en función de un ideal cualquiera» (p. 256). 
La autora también habla de los riesgos potenciales del encuadre interno y en especial refiere a:

el todo vale [...], la facilitación de la sugestión en detrimento de la elaboración, la pérdida de la función del encuadre externo como depositaria de las ansiedades arcaicas (Bleger 1967), la simplificación abusiva, la pérdida de la dosis indispensable de rigor analítico. (Alizade, 2002, p. 14)

Alizade (2002) plantea que el encuadre externo hace posible el trabajo analítico, pero destaca que no puede utilizarse como un ritual a cumplir, pues no reside allí la eficacia del psicoanálisis. A su vez, aclara que el encuadre solo es un impedimento cuando se solidifica en la mente del analista (Alizade, apud Edelman et al., 2009). La autora, al igual que Giovannetti de Freitas (2005), considera que las incertidumbres actuales nos obligan a repensar, flexibilizarnos, atender a las nuevas demandas y utilizar otras habilidades para que se dé el encuentro.

En cuanto a la consideración de la Entrevistada 2 anteriormente citada, se puede pensar que está haciendo referencia a que cada uno de los elementos de la neutralidad debe guardar una relación articulada con los conceptos que darán cuenta del proceso; entre ellos, el encuadre. El psicoterapeuta consultado en la entrevista 3 adhirió a las consideraciones de Schkolnik (2008) al distinguir entre la importancia del establecimiento del encuadre y su cumplimiento como valor protector para ambos protagonistas del proceso psicoterapéutico. Su experiencia lo habilita al siguiente señalamiento:

yo creo que si se genera un encuadre adecuado con el paciente [...] y además cumplirlo es una protección para el paciente y para el terapeuta, muchos no se han dado cuenta de eso, de que uno, que el trabajo nuestro no es un trabajo sencillo. (Entrevistado 3, comunicación personal, 19 de junio, 2018) 
Vale señalar que la mayoría de los entrevistados al hablar del encuadre se remitieron a los parámetros formales y a cómo son planteados al paciente. A su vez, se entiende que cada encuentro es particular y que es posible hacer modificaciones ajustadas que no invalidan el buen desarrollo del proceso psicoterapéutico. Para hacer esas modificaciones ajustadas es imprescindible la formación, entendida en sus tres pilares: formación teórica, análisis personal (reanálisis) y supervisión clínica, indispensable para una segunda mirada. La formación en toda su dimensión es lo que se internaliza y pasa a ser el encuadre interno de cada psicoterapeuta psicoanalítico.

Pues bien, se sabe que el encuadre no es el mismo en sus caracteres formales al de los comienzos del psicoanálisis. Se reconoce también que existen condiciones particulares que permiten el trabajo analítico, que refieren tanto a los aspectos formales como a la internalización de los aspectos que delimitan la actitud analítica. Lo interesante es si se puede pensar en un encuadre estándar para todo tipo de encuentro. ${ }^{7}$ La ética del terapeuta debe estar presente siempre, en el sentido de no manipular al paciente y de respetar sus valores, lo cual se planteó al referirse a la neutralidad analítica. Así que asociar la neutralidad al encuadre supone aceptar, como dicen Laplanche y Pontalis (1996), que es una de las cualidades que definen la posición del analista durante la cura.

7 Se puede investigar la posibilidad de variaciones al encuadre, una de las cuales puede tener relación con el tipo de patología del paciente. Pero también, como se plantea en la tesis, a veces se presentan situaciones en las que la neutralidad hace tope $y$, además de que se exprese el psicoterapeuta, puede ser necesario hacer modificaciones al encuadre, en el entendido de que eso no significa alterar el encuadre interno. 


\section{REFERENCIAS BIBLIOGRÁFICAS}

AlizAde, M. (2002). El rigor y el encuadre interno. Revista Uruguaya de Psicoanálisis, 96, 13-16. https://apuruguay.org/revista_pdf/rup96/ rup96-alizade.pdf

Benítez, L. (2019). Prácticas discursivas acerca de la neutralidad en la clínica actual. Estudio realizado en una asociación uruguaya de psicoterapia [tesis de maestría]. Instituto Universitario de Postgrado de la Asociación Uruguaya de Psicoterapia Psicoanalítica, Uruguay.

Bleger, J. (1967). Psicoanálisis del encuadre psicoanalítico. Revista Argentina de Psicoanálisis, 24(2), 241-258. https://fepal.org/images/ 2002REVISTA/espanol/bleger.pdf

Bleichmar, H. (2013). Lo reprimido, lo no constituido y la desactivación sectorial del inconsciente. Intervenciones terapéuticas diferenciadas. En Avances en psicoterapia psicoanalítica (pp. 117-182). Paidós.

Braun de Bagnulo, S., Altmann de Litvan, M. y Cutinella de Aguiar, O. (1991). Obstáculos clínicos cuestionadores de la función analítica. Revista Uruguaya de Psicoanálisis, 74, 133-146. https://apuruguay. org/apurevista/1990/1688724719917409.pdf

Charmaz, K. C. (2006). Constructing Grounded Theory: A practical Guide Through Qualitative Analysis. SAGE Publications.

Corbella, S. y Botella, L. (2003). La alianza terapéutica: historia, investigación y evaluación. Anales de Psicología, 19(2), 205-221.

www.um.es/analesps/v19/v19_2/04-19_2.pdf

De Urtubey, L. (1999). El encuadre y sus elementos. Revista Uruguaya de Psicoanálisis, 89, 49-67. https://apuruguay.org/apurevista/1990/ 1688724719998904.pdf 
Edelman, L., López, R. y Alizade, M. (agosto, 2009). Cuestionario: Encuadre y dispositivo psicoanalítico. www.topia.com.ar/articulos/ cuestionario-encuadre-y-dispositivo-psicoanal\%C3\%ADtico

EIzIRIK, C. (2002). Entre la objetividad, la subjetividad y la intersubjetividad. ¿Aún hay lugar para la neutralidad analítica? Revista Internacional de Psicoanálisis Aperturas, 012. www.aperturas.org/articulo.php?articulo=0000212

Etchegoyen, R. H. (1986a). Situación y proceso analíticos. En Los fundamentos de la técnica psicoanalítica (pp. 470-478). Amorrortu.

EtCHegoyen, R. H. (1986b). El encuadre analítico. En Los fundamentos de la técnica psicoanalítica (pp. 478-490). Amorrortu.

FREUD, S. (1986a). Dos artículos de enciclopedia: «Psicoanálisis» y «Teoría de la libido» (1923 [1922]). En Obras completas (vol. XviII, pp. 227254). Amorrortu.

FREUD, S. (1986b). Puntualizaciones sobre el amor de transferencia (Nuevos consejos sobre la técnica del psicoanálisis, III) (1915 [1914]). En Obras completas (vol. xII, pp. 161-174). Amorrortu.

FREUD, S. (1986c). Sobre la iniciación del tratamiento (Nuevos consejos sobre la técnica del psicoanálisis, I) (1913). En Obras completas (vol. XII, pp. 121-144). Amorrortu.

GARCíA, S. (2002). Reinterrogando el método psicoanalítico. Revista Uruguaya de Psicoanálisis, 96, 71-87. https://apuruguay.org/revista_pdf/rup98/rup96_garcia.pdf

Giovannetti De Freitas, M. (2005). La hospitalidad, hoy, en la clínica psicoanalítica: interpretación, construcción y deconstrucción. Revista Uruguaya de Psicoanálisis, 100, 254-269. https://apuruguay/revista_pdf/rup100/100-defreitas.pdf

Greben, S. E. y Lesser, S. R. (1976). The question of neutrality in psychotherapy. American Journal of Psychotherapy, 30(4), 623-630. http://doi.org/10.1176/appi.psichotherapy.1976.30.4.623 
Laplanche, J. y Pontalis, J. (1996). Diccionario de psicoanálisis (9. ${ }^{a}$ ed.). Paidós.

MuñIz, M. (2016). De texturas, trama y urdimbre. Revista Uruguaya de Psicoanálisis, 122, 49-57. https://apuruguay.org/apurevista/2010/16887247201612206.pdf

ORMART, E. B. (2005). La neutralidad: de lo imaginario a lo real [ponencia]. XII Jornadas de Investigación y Primer Encuentro de Investigadores en Psicología del Mercosur, Buenos Aires. www.aacademica.org/ 000-051/380.pdf

Ormart, E. B. y Brunetti, J. (2006). Neutralidad: representaciones de los psicoanalistas de la Asociación Psicoanalitica Argentina [ponencia]. XIII Jornadas de Investigación y Segundo Encuentro de Investigadores en Psicología del Mercosur, Buenos Aires. www.aacademica. org/000-039/508

Paciuk, S. (2002). El elogio del encuadre. Revista Uruguaya de Psicoanálisis, 96, 37-56. https://apuruguay.org/revista_pdf/rup96/rup96-paciuk.pdf

RAPPOPORT DE Aisemberg, E. (2004). Ética y neutralidad. Revista de Psicoanálisis, 61(3), 599-605.

Real Academia Española (2021). Encuadrar. Diccionario de la lengua española (23. ${ }^{a}$ ed.) [versión 23.4 en línea]. Recuperado en febrero de 2021. https://dle.rae.es/encuadrar?m=form

ReniK, O. (1999). Los peligros de la neutralidad. Revista Uruguaya de Psicoanálisis, 89, 9-29. https://apuruguay.org/apurevista/1990/ 1688724719998901.pdf

Rosa, J. (1989). Abstinencia y neutralidad. Revista de Psicoterapia Psicoanalitica, 3(1),39-44.www.bvspsi.org.uy/local/TextosCompletos /audepp/025583271989030105.pdf 
Schкolnik, F. (1999). ¿Neutralidad o abstinencia? Revista Uruguaya de Psicoanálisis, 89, 68-81. https://apuruguay.org/apurevista/ 1688724719998905.pdf

Schкolnik, F. (2008). ¿Una práctica psicoanalítica o varias? Revista Uruguaya de Psicoanálisis, 106, 7-21. https://apuruguay.org/apurevista/2000/16887247200810601.pdf

SHAPIRO, T. (2005). Naming the unnameable. Psychoanalytic Inquiry, 25(4), 506-515. https://doi.org/10.2513/s07351690pi2504_6

SCHROEDER, D. (2010). Repensando el encuadre interno. Revista Uruguaya de Psicoanálisis, 110, 140-160. https://apuruguay.org/congresos/2010 /schroeder_damian_2041100_4.pdf

THомÄ, K, y Kächele, H. (1990). Teoría y práctica del psicoanálisis II. Estudios clínicos. Herder. 